\title{
Knowledge, Attitude, Practice and Barriers to Educational Implementation of Non-Pharmacological Pain Management during Labor in Selected Hospitals, Kenya
}

\author{
Poornima Ramasamy ${ }^{1}$, Arthur Kwena ${ }^{2}$, Ashraf Emarah ${ }^{3}$, Simon Kangethe ${ }^{4}$ \\ ${ }^{1}$ School of Nursing, University of Eastern Africa, Eldoret, Kenya \\ ${ }^{2}$ Department of Medical Biochemistry, School of Medicine, Moi University, Eldoret, Kenya \\ ${ }^{3}$ Department of Surgery and Anaesthesiology, School of Medicine, Moi University, Eldoret, Kenya \\ ${ }^{4}$ Department of Medical Education, School of Medicine, Moi University, Eldoret, Kenya
}

Email address:

poorniramasamy@gmail.com(P. Ramasamy), arthurkwena@gmail.com(A. Kwena), ashrafemarah@gmail.com(A. Emarah), simonkangethe12@gmail.com(S. Kangethe)

\section{To cite this article:}

Poornima Ramasamy, Arthur Kwena, Ashraf Emarah, Simon Kangethe. Knowledge, Attitude, Practice and Barriers to Educational Implementation of Non-Pharmacological Pain Management During Labor in Selected Hospitals, Kenya. Central African Journal of Public Health. Vol. 4, No. 1, 2018, pp. 20-26. doi: 10.11648/j.cajph.20180401.14

Received: February 12, 2018; Accepted: March 5, 2018; Published: March 30, 2018

\begin{abstract}
A Study to Evaluate the Effectiveness of knowledge, attitude, practice and barriers to implementation of non-pharmacological pain management during labor using non-experimental cross sectional descriptive study design and used a structure questionnaire to collect data from a sample of the population. The majority of the health care providers' have inadequate knowledge regarding non-pharmacological pain management during labor. Health care providers have a positive attitude towards non-pharmacological pain management during labor. Most of the health care providers practice massaging, breathing exercise and encourage different laboring positions during labor. The main barriers were lack of time, lack of knowledge and patient unwillingness.
\end{abstract}

Keywords: Health Care Providers, Knowledge, Non-Pharmacological, Pain Management During Labor

\section{Introduction}

Labor and birth are enormous emotional and physiologic accomplishments in life. It is one of the most marvelous and memorable segment in a woman's life. It does not really matter if the child is first, second or the third one. Each experience is unique and calls for a celebration (Dawley, 2000). The world Health Organization (WHO, 1997) defines normal labor as low risk throughout, spontaneous in onset with fetus presenting by the vertex, culminating in the mother and infant in good condition following birth. During the process of giving birth to new life, the mother has to undergo enormous pain. Labor pain is an unpleasant, complex, highly individualized phenomenon with both sensory and emotional components. Childbirth while primarily a joyful event predisposes the mother to one of the most severe forms of pain ever reported (Sr. Barthalomea Joseph, 2012). For several decades, childbirth educators have focused on the alleviation or reduction of pain and suffering during the childbearing experience. A wide array of non-pharmacological pain relief measures, as well as pharmacological interventions, is presently available to women in labor. Relaxation, breathing techniques, positioning/movement, massage, hydrotherapy, hot/cold therapy, music, guided imagery, acupressure, and aromatherapy are some self-help comfort measures women may initiate during labor to achieve an effective coping level for their labor experience (Nichols and Humenick, 2000). Non-pharmacological or natural therapies are methods that help decrease the pain. These therapies do not involve taking medicines. People have used "natural" ways to help with pain and healing from the very beginning of time. Non-pharmacologic interventions include cognitive behavioral therapy, relaxation therapy, biofeedback, patient education, self-management, and social support interventions. These types of interventions aim to change behavior, 
cognitions, and emotions by targeting the psychosocial processes that are implicated in the perceptions and response to pain. There is good evidence that these interventions can be effective in managing pain, particularly in relation to the cognitions surrounding pain; this, however, is predominantly in the short term (Demir, 2012; Reuters, 2012; James et al., 2011). The health care providers should have knowledge of the use of non-pharmacological approaches such as the use of hot and cold mechanisms, acupuncture, massages and breathing measures among others employed in pain management. In addition to the areas of pain management required of a nurse, knowledge of the existing standards of pain management as well as the already established recommendations is considered to be vital, as nurses have the potential to play a vital role in pain management and education. A major prerequisite of effective care delivery is nurses who are prepared at a fundamental level of current knowledge, competence and confidence in understanding and managing pain. A pathway is created with the development of this knowledge and a skills framework which will promote consistent evidence-based practice and will thus contribute to improved health outcomes for the sufferer/s (King, 2011; Kipkorir, 2011). Childbirth has a deep significance not only to mother and her partner but also to the whole family. It is a profound physiological, psychosocial and spiritual event. It is this joy and expectation from the entire family that drives the mother to face the pain and all the suffering associated with it (Sr. Barthalomea Joseph, 2012). The management of labor pain is one of the main goals of maternity care. Although pain and suffering occur together, one may suffer without pain or have pain without suffering. The goal of eliminating labor pain not only requires pain medication but also require other techniques/modalities to preserve health and maintain coping strategies. This requires highly skilled personnel to control any undesirable side effects. The effectiveness of most widely used alternative modalities is discussed with a view to educate the primary care providers, the midwives (Bolding and Simkin, 2004). The investigator's previous clinical experience and also many studies conducted in different settings show that alternative modalities are effective to relieve pain during labor. With the effectiveness of various alternative modalities proved by different studies, the investigator wants to assess the knowledge, attitude, and practice and to find out the barriers that prevent the health care providers from using non pharmacological pain management during labor. The birthing process has evolved considerably over time. The cesarean section rate rose $60 \%$ between 1996 to $32.9 \%$ in 2009 , but are currently remaining steady around $32.7 \%$ in 2013 (Hamilton, Martin, Osterman, \& Curtin, 2014, p. 7). This change over a thirteen year time span is drastic and shows how childbirth has devolved from a natural, healthy aspect of womanhood, into a medicalized process that requires intervention. Birth plans are often disregarded when mothers enter the hospital setting and surrender to the medical version of childbirth. Too often mothers are being treated with increased interventions and epidurals instead of being coached and empowered to cope with the pain. In order to reverse this medicalized child birth trend, the current issues need to be clearly identified.

\section{Method}

\subsection{Study Design}

This study employed a non-experimental cross-sectional descriptive survey design and used a structured questionnaire to collect data from a sample of the population at a specific point in time and determined relationships among variables of interest.

\subsection{Study Site}

This study was conducted in two medical institutions providing health care services in Moi Teaching and Referral hospital (MTRH) and Jaramogi Oginga Odinga Teaching and Referral Hospital, Kenya.

\subsection{Study Population and Sample}

The target population for this study was all health care providers in Moi Teaching and Referral Hospital and Jaramogi Oginga Odinga Teaching and Referral Hospital. The accessible population was 286 of staff nurses - 68, student nurses -195 , resident -1 , medical officers -9 , intent -3 and clinical officers - 10 .

\subsubsection{Inclusion Criteria}

The study included all health care providers and maternity student nurses working in the selected two hospitals and who fulfill the inclusion criteria.

i. Health care providers working in labor ward.

ii. Student nurses that are studying the maternity course and are then posted in the same.

iii. Health care providers and student nurses who are willing to participate in the study.

\subsubsection{Exclusive Criteria}

i. Health care providers who are not available during data collection period.

ii. Those who are on annual leave.

\subsection{Sampling Techniques and Sampling Procedure}

A census sampling was used to gather the information from the health care providers regarding non-pharmacological pain management during labor. Self-administered questions on knowledge, attitude, practice and barriers regarding non-pharmacological pain management during labor were given to the health care providers who were asked to complete the self-administered questions during their break time (30-40 minutes), so as not to interrupt patient care and other daily duties.

\subsection{Data Collection Instruments}

The instrument used in this study is a self-structured questionnaire which was compiled by the researcher by using review of literature and the text books.

Section A: Demographic data of the respondents. 
Section B: Consist of parts I to XII, which are knowledge questions on general information regarding labor, non-pharmacological pain management during labor like Aromatherapy, Massage, Breathing Exercise, Inter-dermal water blocks, Hydrotherapy, Continuous labor support, Movement and Positioning, Acupressure, Health and Cold application and Music therapy.

Section C: Three point "Likert scale" for measuring the respondents attitude through statements will be used.

Section D: Check list was used to determine the practice of the health care providers on non-pharmacological pain management during labor.

Section E: Check list was used to determine the barriers for using non-pharmacological pain management during labor.

\subsection{Data Analysis Procedures}

The data in the questionnaires were to first cleaned by removing those that are not correctly filled in, especially section A that deals with the demographic data of the participants including: age, gender, position, etc. After data cleaning, the remaining questionnaires were subject to further analysis. The completed coded data was entered into the computer using Statistical Package for Social Sciences (SPSS Version 21.0). Descriptive statistics options are mainly utilized to analyze the data into mean, median, mode and standard deviation. The results from this study were presented in frequency distribution tables, Pearson product moment correlation coefficient and nonparametric statistical procedure including the Chi-Square to be used to determine of significance/relationship and determine if the responses are similar among health care providers in different forms.

\subsection{Validity and Reliability}

\subsubsection{Validity}

After constructing the questionnaire by modifying it based on review of recent literature, the researcher sought opinion from the supervisors with extensive experience in medical and nursing practice and training to ascertain whether the questionnaire was indeed relevant. Feedback from the supervisors and the recommendation from IREC was incorporated into the questionnaire and corrections made accordingly.

\subsubsection{Reliability}

A test-retest reliability coefficient is obtained by administering the same test on two different occasions, then correlating the scores obtained on each occasion.

The researcher administered the test to a group of 50 health care providers in County General Hospital, Kakamega (for the pilot study) within the duration of two weeks. The correlation coefficient was calculated based on the two sets of scores obtained from this population. The correlation coefficient was found to be 0.84 . Lee recommends that correlation values higher than 0.80 are sufficient to qualify the test as reliable.

\section{Result: Data Presentation, Analysis and Interpretation}

\subsection{Combined Responses for Demographic Data}

The demographic characteristics of the health care providers have been described according to their age, gender, highest qualification, current position and year of experience in labor ward.

\subsubsection{Age of the Respondents in Years}

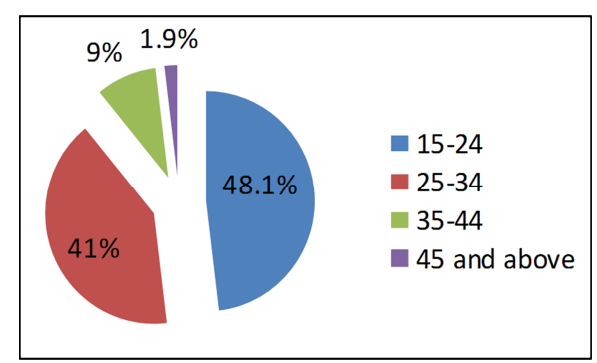

Figure 1. Age of the Respondents in Years.

The age groups of the participants were as follows: $41 \%$ of the participants were aged between 25 to 34 years, $48.1 \%$ of the participants were 15 to 24 years, $9 \%$ of the participants were 35 to 44 years and $1.9 \%$ of the respondents were over the age of 45 years.

\subsubsection{Gender of the Participants}

The gender of the participants comprised $63.2 \%$ females and $36.8 \%$ males.

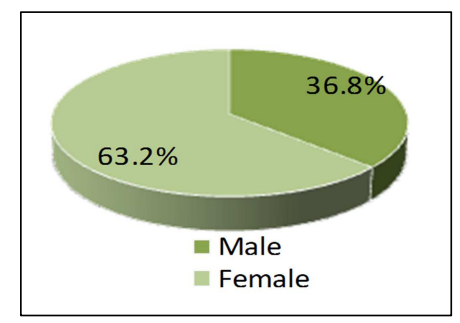

Figure 2. Gender of the Participants.

\subsubsection{Current Position of the Participants}

Current position of the participants as follows: $67.9 \%$ of the participants were student nurse/Midwife, $25.6 \%$ of the participants were nurse/midwife, $3.4 \%$ of the participants were medical officers, $2.3 \%$ of the participants were the House/Clinical officer, $0.4 \%$ of the participants were Residential Doctor and $0.4 \%$ of the participant was Consultant.

\subsubsection{Any Additional Training in Non-Pharmacological Pain Management during Labor}

Eighty three point eight percent of the participants responded that they did not have any additional training in non-pharmacological pain management during labor. Fifteen point four percent of participants responded that they had gone for the training while $0.8 \%$ of the respondents did not show 
any response.

\subsubsection{If Yes Mention the Year and Types of Training}

Among 266 participants two nurse midwives had had a special training on first aid including pain management in labor and importance of using non-pharmacological pain management. One clinical officer had a seminar on Massage. One nurse had attended a seminar on distraction.

\subsubsection{Years of Experience}

Seventy six point seven percent of the respondents have 0 to 5 years' experience, $13.5 \%$ of the respondents have 6 to 10 years of experience, $3.8 \%$ of the respondents have 11 to 15 years of experience, $2.6 \%$ of the respondents have above 16 years of experience and $3.4 \%$ of the respondents did not respond.

\subsubsection{Non-Pharmacological Education is needed}

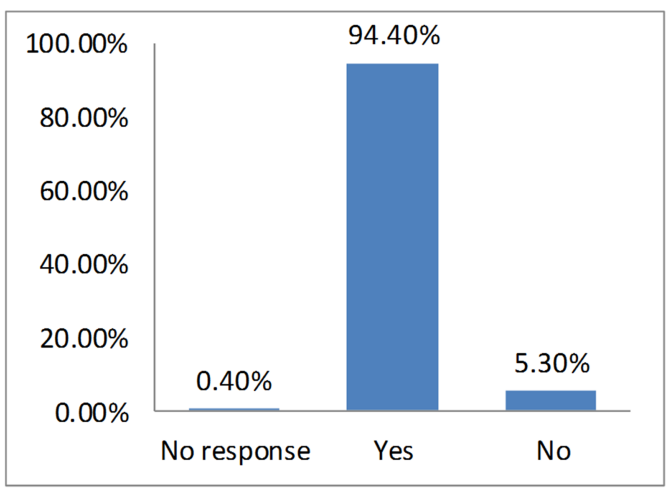

Figure 3. Non-Pharmacological Education is needed.

\subsection{Level of Health Care Providers Knowledge Regarding Non-Pharmacological Pain Management during Labor}

1. To assess the knowledge of the health care providers towards the non-pharmacological pain management during labor.

Overall Knowledge of non-pharmacological pain management during labor was $42.40 \%$. Among 266 of the participants, knowledge of general information regarding labor is $53.52 \%$, knowledge of non-pharmacological pain management during labor is $65.04 \%$, knowledge of aromatherapy is $20.93 \%$, knowledge of massage is $40.23 \%$, knowledge of breathing exercises is $15.04 \%$, knowledge of intra-dermal water blocks is $37.97 \%$, knowledge of hydrotherapy is $40.85 \%$, knowledge of continuous labor support is $58.40 \%$, knowledge of movement and positioning is $50.75 \%$, knowledge of acupressure is $26.32 \%$, knowledge of heat and cold is $27.44 \%$ and knowledge of music and audio analgesia is $54.89 \%$.

\subsection{Level of Attitude towards Non-Pharmacological Pain Management during Labor}

To identify the attitude of the health care providers towards the non-pharmacological pain management during labor.

\subsubsection{Does Aromatherapy Help to Reduce the Level of Labor Pain}

Fifty nine point eight $(59.8 \%)$ of the respondents agreed that aromatherapy help to reduce the level of labor pain.

\subsubsection{Do you Think Massage Helps in Release of Endorphine}

Fifty five point six $(55.6 \%)$ of the respondents agreed that massage helps in and release of endorphine.

\subsubsection{Will Back Massage Warm up Women in Labor}

Interestingly $60.5 \%$ of the respondents agreed that back massage warm up women in labor.

\subsubsection{Do you Think Breathing Exercise Helps the Baby to Get More Oxygen}

It is also noteworthy that most of the respondents $66.2 \%$ agreed that breathing exercise helps the baby to get more oxygen.

\subsubsection{Would you recommend the Breathing Exercise During Labor}

Seventy nine point seven of the respondents agreed that they would recommend the breathing exercise during labor.

\subsubsection{Do you Think Hydrotherapy Helps During Labor to Reduce Pain}

Forty seven point seven percent of the respondents agreed that hydrotherapy helps to reduce labor pain.

\subsubsection{Does Water Immersion Help in Increased Circulation}

Forty nine point six percent of the respondents agreed that water immersion help in increased circulation.

\subsubsection{Do you Think Heat and Cold Application Helps in Reducing the Labor Pain}

Fifty six percent $(56.0 \%)$ of the respondents agreed that heat and cold application helps in reducing labor pain.

\subsubsection{Would you Recommend Continuous Labor Support}

Sixty one point three percent of the respondents agreed that they will recommend continuous labor support during labor.

\subsubsection{Does Acupressure Help Muscle Fibres Elongate and Relax Allowing Blood to Flow More Freely}

Forty nine point six percent of the respondents agreed that the acupressure help muscle fibers elongate and relax allowing blood to flow more.

\subsubsection{Do you Think Semi Sitting Position during Labor Is Not Comfortable}

Forty point six percent of the respondents agreed that semi sitting position during labor is not comfortable. Sixty eight point eight percent of the respondents agreed that they would recommend squatting position during labor.

\subsubsection{Does Music Therapy Help to Reduce the Level of Labor Pain}

Interestingly, seventy one point eight percent of the respondents agreed that music therapy helps to reduce the level of labor pain. Forty six point two percent of the respondents agreed that music therapy helps to maintain heart rate and blood pressure. 


\subsection{Level of Practice of Non-Pharmacological Pain Management during Labor}

1. To determine the practice of health care providers towards non-pharmacological pain management during labor.

Table 1. I use Aromatherapy during labor.

\begin{tabular}{llllll}
\hline & & Frequency & Percent & Valid Percent & Cumulative Percent \\
\hline \multirow{4}{*}{ Valid } & NO RESPONSE & 16 & 6.0 & 6.0 & 6.0 \\
& NEVER & 196 & 73.7 & 73.7 & 79.7 \\
& SOMETIMES & 45 & 16.9 & 16.9 & 96.6 \\
& ALWAYS & 9 & 3.4 & 3.4 & 100.0 \\
& Total & 266 & 100.0 & 100.0 & \\
\hline
\end{tabular}

Table 2. I massage the laboring mother.

\begin{tabular}{llllll}
\hline & & Frequency & Percent & Valid Percent & Cumulative Percent \\
\hline \multirow{4}{*}{ Valid } & NO RESPONSE & 16 & 6.0 & 6.0 & 6.0 \\
& NEVER & 15 & 5.6 & 5.6 & 11.7 \\
& SOMETIMES & 102 & 38.3 & 38.3 & 50.0 \\
& ALWAYS & 133 & 50.0 & 50.0 & 100.0 \\
& Total & 266 & 100.0 & 100.0 & \\
\hline
\end{tabular}

Table 3. I encourage mothers to do breathing exercise during labor.

\begin{tabular}{llllll}
\hline & & Frequency & Percent & Valid Percent & Cumulative Percent \\
\hline \multirow{4}{*}{ Valid } & NO RESPONSE & 14 & 5.3 & 5.3 & 5.3 \\
& NEVER & 11 & 4.1 & 4.1 & 9.4 \\
& SOMETIMES & 35 & 13.2 & 13.2 & 22.6 \\
& ALWAYS & 206 & 77.4 & 77.4 & 100.0 \\
& Total & 266 & 100.0 & 100.0 & \\
\hline
\end{tabular}

Table 4. I use intradermal water block during labor.

\begin{tabular}{llllll}
\hline & & Frequency & Percent & Valid Percent & Cumulative Percent \\
\hline \multirow{4}{*}{ Valid } & NO RESPONSE & 15 & 5.6 & 5.6 & 5.6 \\
& NEVER & 188 & 70.7 & 70.7 & 76.3 \\
& SOMETIMES & 46 & 17.3 & 17.3 & 93.6 \\
& ALWAYS & 17 & 6.4 & 6.4 & 100.0 \\
& Total & 266 & 100.0 & 100.0 & \\
\hline
\end{tabular}

Table 5. I encourage and use hydrotherapy during labor.

\begin{tabular}{llllll}
\hline & & Frequency & Percent & Valid Percent & Cumulative Percent \\
\hline \multirow{4}{*}{ Valid } & NO RESPONSE & 19 & 7.1 & 7.1 & 7.1 \\
& NEVER & 170 & 63.9 & 63.9 & 71.1 \\
& SOMETIMES & 58 & 21.8 & 21.8 & 92.9 \\
& ALWAYS & 19 & 7.1 & 7.1 & 100.0 \\
& Total & 266 & 100.0 & 100.0 & \\
\hline
\end{tabular}

Table 6. I would always ensure women's relatives are with laboring Women during the entire labor process.

\begin{tabular}{llllll}
\hline & & Frequency & Percent & Valid Percent & Cumulative Percent \\
\hline \multirow{4}{*}{ Valid } & NO RESPONSE & 17 & 6.4 & 6.4 & 6.4 \\
& NEVER & 19 & 7.1 & 7.1 & 13.5 \\
& SOMETIMES & 122 & 45.9 & 45.9 & 59.4 \\
& ALWAYS & 108 & 40.6 & 40.6 & 100.0 \\
& Total & 266 & 100.0 & 100.0 & \\
\hline
\end{tabular}

Table 7. I always use different positions according to the need of the laboring women.

\begin{tabular}{llllll}
\hline & Frequency & Percent & Valid Percent & Cumulative Percent \\
\hline \multirow{4}{*}{ Valid } & NO RESPONSE & 17 & 6.4 & 6.4 & 6.4 \\
& NEVER & 20 & 7.5 & 7.5 & 13.9 \\
& SOMETIMES & 96 & 36.1 & 36.1 & 50.0 \\
& ALWAYS & 133 & 50.0 & 50.0 & 100.0 \\
& Total & 266 & 100.0 & 100.0 & \\
\hline
\end{tabular}


If you Support Different Positions Kindly Mention the Position you Use for the Laboring Women

Forty percent of the respondents encouraged squatting position for laboring women and $30 \%$ used standing and squatting position. Twenty percent of the respondents encouraged knee chest, side lying, standing and lithotomy positions.

Table 8. I give acupressure during labor process.

\begin{tabular}{llllll}
\hline & & Frequency & Percent & Valid Percent & Cumulative Percent \\
\hline \multirow{4}{*}{ Valid } & NO RESPONSE & 17 & 6.4 & 6.4 & 71.1 \\
& NEVER & 189 & 49 & 71.1 & 18.4 \\
& SOMETIMES & 11 & 18.4 & 4.1 & 77.4 \\
& ALWAYS & 266 & 100.0 & 100.0 \\
\hline
\end{tabular}

Table 9. I do warm fomentation for labor women to relieve her pain.

\begin{tabular}{llllll}
\hline & & Frequency & Percent & Valid Percent & Cumulative Percent \\
\hline \multirow{4}{*}{ Valid } & NO RESPONSE & 12 & 4.5 & 4.5 & 6.5 \\
& NEVER & 169 & 67 & 63.5 & 68.5 \\
& SOMETIMES & 18 & 25.2 & 25.2 & 6.8 \\
& ALWAYS & 266 & 100.0 & 93.2 & 100.0 \\
\hline
\end{tabular}

Table 10. I use ice packs during labor for decreasing the labor pain.

\begin{tabular}{|c|c|c|c|c|c|}
\hline & & Frequency & Percent & Valid Percent & Cumulative Percent \\
\hline \multirow{5}{*}{ Valid } & NO RESPONSE & 13 & 4.9 & 4.9 & 4.9 \\
\hline & NEVER & 177 & 66.5 & 66.5 & 71.4 \\
\hline & SOMETIMES & 61 & 22.9 & 22.9 & 94.4 \\
\hline & ALWAYS & 15 & 5.6 & 5.6 & 100.0 \\
\hline & Total & 266 & 100.0 & 100.0 & \\
\hline
\end{tabular}

Table 11. I encourage the laboring women to listen to soothing music during labor.

\begin{tabular}{llllll}
\hline & & Frequency & Percent & Valid Percent & Cumulative Percent \\
\hline \multirow{4}{*}{ Valid } & NO RESPONSE & 12 & 4.5 & 4.5 & 3.5 \\
& NEVER & 94 & 35.3 & 35.3 & 44.4 \\
& SOMETIMES & 118 & 44.4 & 15.8 & 84.2 \\
& ALWAYS & 42 & 15.8 & 100.0 \\
\hline
\end{tabular}

\subsection{Barriers towards Non-Pharmacological Pain Management during Labor}

1. To assess the barriers to non-pharmacological pain management during labor.

\subsubsection{Health-Care System Related Barriers}

In terms of health care system related barriers among 266 participants, 254 participants responded that the main barrier was lack of time, 246 participants responded that their barrier was regulatory issues (policy), 247 participants responded that their barrier was inadequate staff members and 246 participants responded that their barrier was lack of equipment.

\subsubsection{Health-Care Provider Related Barriers}

In health care provider related barriers among 266 participants, 249 participants responded that the main barrier was Dr./RN unwillingness, 248 participants responded that lack of knowledge; 242 responded that it is difficult to apply; 244 participants responded that they need critical thinking; 244participants responded that it's hard to measure and 242 participants responded that the barrier was efficacy.

\subsubsection{Patient Related Barriers}

In patient related barriers among 266 participants; 251 participants responded that the main barrier was patient unwillingness; 242 participants responded that it is sometimes not as concrete; 249 participants responded that strong beliefs of analgesia; 249 participants responded that pain was too severe; 246 participants responded that they needed build to trust and 251 participants responded that it depended on the age of the client.

\section{Discussion}

From this study, health care providers have inadequate knowledge regarding non-pharmacological pain management during labor. But when coming to their level of education for the undergraduate students, non-pharmacological pain management is not elaborated in their curriculum that makes their practice less effective during labor for the laboring mother, whereas for the postgraduate students they do an elaborate study about non-pharmacological pain management during labor but coming to the point of practicum they do lesser work when compare with undergraduate. 


\section{Conclusion}

The majority of the health care providers' comprising $42 \%$ has inadequate knowledge regarding non-pharmacological pain management during labor. Health care providers have a positive attitude towards non-pharmacological pain management during labor. Most of the health care providers practice massaging, breathing exercise and encourage different laboring positions during labor. The main barriers were lack of time, lack of knowledge and patient unwillingness. The management of mother's pain in labor is uncomprehensive.

\section{References}

[1] Bolding, A., \& Simkin, P. (2004). Update on non-pharmacological approaches to relieve pain and prevent suffering. British Journal of Obstetrics and Gynaecology, 49 (6), 489-504.

[2] Dawley, K. (2000). The campaign to eliminate the midwife. The American Journal of Nursing. 100 (10), 50-56.

[3] Demir, Y. (2012). Non-Pharmacological Therapies in Pain Management, Pain Management - Current Issues and Opinions. Gabor B. Racz and Carl E. Noe (Ed.).

[4] Hamilton, B. E., Martin, J. A., Osterman, M. J., \& Curtin, S. C. (2014). Births: Preliminary data for 2013. National Vital
Statistics Reports web release; vol 63 no 01, National Center for Health Statistics, Centers for Disease Control and Prevention, Hyattsville, MD. Retrieved from Centers for Disease Control and Prevention.

[5] James, H., Hochberg, M., Silman, A., Smolen, J., Weinblatt, M., \& Weisman, M. (2011). Non-pharmacologic pain management: Rheumatology. 5ed, Elsevier, Philadelphia, USA, ISBN: 978-0-323-06551-1.

[6] King, S. (2011). New Zealand pain management nursing knowledge and skills framework. New Zealand pain society.

[7] Kipkorir, C. (2011). Knowledge and attitudes of nurses towards pain management among the elderly: a case study of registered nurses from the local healthcare centres, Kokkola. M. S. thesis Central Ostrobothnia University. Faculty of Applied Sciences. p. 60 .

[8] Nichols, F. \& Humenick, S. (2000), Childbirth education: Practice, research and theory, 2nd edition editors. Philadelphia (PA): WB Saunders Company.

[9] Reuters, T. (2012). Non-pharmacological Pain Management Therapies For

Adults.http://www.drugs.com/cg/non-pharmacologicalpain-ma nagement-therapies-for-adults.html (accessed 25-3-2012).

[10] Sr. Barthalomea Joseph. (2012). A study to assess the effectiveness of acupressure on labor in full term gravid women in selected hospitals, Mangalore. Unpublished thesis submitted to RGUHS. 